\title{
Análise do custo e do potencial de geração de energia fotovoltaica em uma universidade pública brasileira
}

\section{Josiane Tamires Santos Silva ${ }^{1}$, Janaina Kawata de Alencar ${ }^{1}$, Sandra Cristina de Oliveira ${ }^{2}$, , Sérgio Silva Braga Júnior $^{2}$ e Angélica Gois Morales ${ }^{2}$}

\begin{abstract}
${ }^{1}$ Universidade Estadual Paulista. Faculdade de Ciências e Engenharia. Programa de Pós-Graduação em Agronegócio e Desenvolvimento. Campus de Tupã. Rua Domingos da Costa Lopes, 780. Jd. Itaipu. Tupã-SP, Brasil (CEP 17602-496).

${ }^{2}$ Universidade Estadual Paulista. Faculdade de Ciências e Engenharia. Departamento de Gestão, Desenvolvimento e Tecnologia. Programa de PósGraduação em Agronegócio e Desenvolvimento. Campus de Tupã. Rua Domingos da Costa Lopes, 780. Jd. Itaipu. Tupã-SP, Brasil (CEP 17602-496). *E-mail: sandra.oliveira@unesp.br.
\end{abstract}

Resumo. As fontes de energia limpas e renováveis como a energia solar têm ganhado destaque pelo seu potencial em termos de sustentabilidade. Assim, o objetivo deste trabalho é estimar o custo e o potencial de geração de energia elétrica fotovoltaica nos campi da Universidade Estadual Paulista (UNESP). Utilizou-se a técnica estatística de análise de clusters associada à de análise de variância (ANOVA), considerando os 24 municípios-sede desses campi, a fim de agrupar aqueles com características similares de incidência solar e de altitude. Foram obtidos quatro clusters distintos, e selecionou-se, de cada cluster, o campus com a menor unidade de área construída, para aferição do custo de implantação e do potencial de captação de energia de um sistema por painéis fotovoltaicos. Foram considerados seis modelos de placas fotovoltaicas com os seguintes atributos: modelo, dimensões, peso, potência, eficiência, tipo de célula e preço. Observou-se que a placa CS6K300MS apresenta um desempenho de captação solar mais eficiente, não requer uma quantidade muito grande de placas, e o custo é mediano em relação às demais. Ainda que a aferição tenha sido feita para o campus com menor área construída, dentro de cada cluster, pode-se prever um planejamento e resultados análogos, em termos de custo e de geração de energia, para os outros campi pertencentes ao mesmo cluster. Por fim, ressalta-se a metodologia proposta, pois tem potencial para ser considerada ou expandida a empreendimentos similares, de forma que um planejamento minucioso seja realizado para a aquisição e instalação desse sistema, e seja garantida a sua viabilidade.

Recebido $06 / 12 / 2019$

Aceito $27 / 02 / 2020$

Disponível on line $08 / 03 / 2020$

Publicado $30 / 04 / 2020$

Acesso aberto

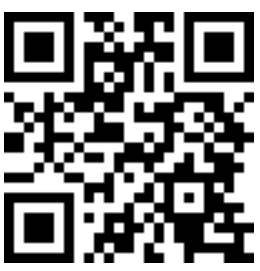

ORCID

D 0000-0001-9822-5440 Josiane Tamires Santos Silva 
Palavras-chave: Energia limpa e renovável; Fotovoltaico; Sustentabilidade; Análise de agrupamentos.

Abstract. Cost and potential analysis of photovoltaic power
generation at a Brazilian public university. Clean and renewable energy sources, such as solar energy, have stood out for their potential. So, the aim of this work is to estimate the cost and potential of photovoltaic power generation on São Paulo State University (UNESP) campuses. The statistical technique of cluster analysis associated with the analysis of variance (ANOVA) was used, considering the 24 host cities of these campuses, in order to form groups of campuses with similar characteristics of solar incidence and altitude. Four distinct clusters were formed, and the campus with the smallest built-up area was selected within each cluster to measure the cost of deployment and the potential for energy capture of a system by photovoltaic panels. Six photovoltaic plate models with attributes such as model, dimensions, weight, power, efficiency, cell type, and price were considered for the study. It was observed that the CS6K300MS plate presents a more efficient solar capture performance, does not require a very large number of plates, and the cost is median in relation to the others. Although the calculations presented have been made for the campus with the smallest built-up area within each cluster, similar cost and power generation planning and results can be expected for the other campuses within the same cluster. Finally, it is emphasized that the proposed methodology has the potential to be considered or expanded to similar enterprises so that thorough planning is carried out for the acquisition and installation of this system, and the viability of the enterprise is guaranteed.

Keywords: Clean and renewable energy; Photovoltaic; Sustainability; Clusters analysis.
(ㄱ) 0000-0001-7771-3293

Janaina Kawata de

Alencar

D) 0000-0002-0968-0108

Sandra Cristina de

Oliveira

D) 0000-0002-4979-1988

Sérgio Silva Braga Júnior

0000-0003-0756-2148

Angélica Gois Morales

\section{Introdução}

Ao longo da história, a humanidade vem se desenvolvendo e evoluindo, sendo notável a ocorrência de mudanças estruturais e organizacionais de diversos setores. Podese dizer que esses avanços tiveram origem com o surgimento da matemática, da geometria e da engenharia (Farias e Sellitto, 2011). 0 desenvolvimento dessas ciências tornou-se ainda mais significativo no século XVIII, com o advento da Revolução Industrial. 0 surgimento da máquina a vapor desencadeou uma série de novas descobertas relativas ao uso de novas e diversas fontes de energia. Nesse sentido, é válido salientar que, até então, a energia utilizada em todas as atividades provinha da força humana, da força animal e de quedas d'água (Lopez, 2012).

Ora estando os avanços tecnológicos cada vez mais aprimorados a partir do século XIX, passou-se então a utilizar a energia elétrica, que foi empregada em diversos setores. Inicialmente era gerada da queima de carvão mineral e, posteriormente, também do petróleo (Farias e Sellitto, 2011; Lopez, 2012). Desde então, a vida humana tornou-se mais confortável, mas ao mesmo tempo dependente do uso da energia elétrica. 
Com o passar dos tempos, diversas fontes passaram a ser exploradas para a geração de energia elétrica, dividindo-se em fontes renováveis e não renováveis. De acordo com Reis (2015), caracterizam-se por fontes não renováveis aquelas passíveis de se esgotarem por serem utilizadas com velocidade bem maior que os milhares de anos necessários para sua formação. Ainda segundo o autor, nesse grupo estão o petróleo e seus derivados, a energia geotérmica e o gás natural. Já as fontes renováveis correspondem àquelas que a natureza consegue repor em menor espaço de tempo do que sua utilização, que é o caso da água, das marés, do sol e dos ventos, além da biomassa e outras (Reis, 2015).

No decorrer dos séculos e ao longo do desenvolvimento da humanidade, pouca ou nenhuma atenção esteve voltada para a finitude dos recursos não renováveis. Posto isso, chegou-se à era da globalização com uma imensa demanda de energia elétrica, mas com escassez de recursos naturais para a geração de energia. Contudo, hoje, versa-se sobre a geração de energia elétrica por meios alternativos e sustentáveis. Segundo Kunz et al. (2018), os países desenvolvidos dispõem de maiores recursos, possibilitando o poder de paridade entre sua população, cenário esse que os países em desenvolvimento não conseguem alcançar. Logo, deduz-se que a garantia de eletricidade influencia diretamente no desenvolvimento econômico e populacional dos países.

No Brasil, a matriz energética baseia-se na geração hidroelétrica, ainda que haja também outras fontes geradoras no país. Por possuir grandes reservas de recursos naturais, o Brasil utiliza seu potencial hidroelétrico para gerar energia limpa e renovável. Apesar de ser considerada energia limpa e renovável, a geração hidroelétrica causa, quando da sua instalação, grandes problemas ambientais, sociais e culturais, como alagamentos, perda de biodiversidade, desapropriação de áreas indígenas, comunidades ribeirinhas, perda da identidade cultural, etc. (Borges e Zouain, 2011; Kunz et al., 2018).

Tanto no panorama nacional como no mundial, a demanda por energia elétrica vem aumentando significativamente, pressionando a sociedade para que busque novas formas de produzir energia para suprir a crescente demanda mundial, porém, sem agredir a natureza (Lopez, 2012; Pereira et al., 2006). Assim, a geração de eletricidade por meio da luz solar, os chamados painéis fotovoltaicos, se apresenta como uma alternativa, produzindo energia limpa, até então, de fonte inesgotável e principalmente sem poluição (Silva et al., 2017).

De acordo com a Secretaria de Energia do Estado de São Paulo (São Paulo, 2006), o Brasil (em especial o Estado de São Paulo) apresenta um cenário privilegiado para a produção de energia solar, tendo em vista sua extensão territorial e seu fator de incidência solar durante todo o ano. No entanto, o aproveitamento de energia solar ainda é incipiente.

Dado o contexto apresentado, o objetivo deste trabalho é estimar o custo e o potencial de geração de energia elétrica fotovoltaica nos campi da Universidade Estadual Paulista (UNESP), buscando contribuir para a elaboração de políticas públicas que possam fomentar a implementação de geração de energia por painéis fotovoltaicos, a fim de reduzir custos de consumo de energia, bem como contribuir para o desenvolvimento socioeconômico das localidades de instalação da universidade.

Resultante da integração de vários institutos isolados de ensino superior instalados em diversas localidades do estado de São Paulo, e de acordo com a Lei 952 de 30 de janeiro de 1976, fundou-se a UNESP, tendo sido nomeada "Júlio de Mesquita Filho". Em 1977, sediada temporariamente em São Paulo, a UNESP dispunha de 14 campi.

O processo de expansão tornou-se possível no decorrer dos anos, por meio de diferentes cenários de desenvolvimento econômico e político. Nos anos de 1990 expandiu-se a oferta de vagas e, posteriormente, em 2003, o governo estadual expandiu a sua área de atuação com a criação de oito novas unidades, localizadas nos municípios de Dracena, Itapeva, Ourinhos, Registro, Rosana, São Vicente, Sorocaba e Tupã. Inicialmente chamadas 
de Unidades Diferenciadas e, a partir de 2006, denominadas de Campi Experimentais (CE). Entre os anos de 2012 e 2015 foram criados onze novos cursos de Engenharia, e a cada ano a UNESP vem inovando e crescendo. Atualmente conta com 34 campi, distribuídos em 24 municípios do estado de São Paulo, sendo 22 no interior, um na capital e um no litoral paulista, que ofertam conjuntamente 183 diferentes cursos de graduação em 60 carreiras de nível superior (Massini-Cagliari et al., 2018).

\section{A energia solar fotovoltaica}

Define-se por energia solar fotovoltaica a conversão de energia solar em eletricidade, e esse processo ocorre por meio de painéis compostos por células fotovoltaicas compostas de materiais semicondutores. A base para a produção de células fotovoltaicas é o silício cristalino (Silva, 2015).

De acordo com Machado e Miranda (2014), a palavra "fotovoltaico" surgiu na França em 1839, quando o físico francês Edmond Becquerel observou a produção de eletricidade em placas de latão imersas em eletrólitos líquidos expostas à luz solar. Assim, surgiu a ideia de produção de energia elétrica por meio da luz solar. Em 1883, o inventor americano Charles Fritts desenvolveu a primeira bateria solar. Anos mais tarde, em 1954, cientistas criaram a primeira célula solar à base de silício. No mesmo ano, segundo os mesmos autores, o jornal americano The New York Times anunciou que as células fotovoltaicas de silício poderiam ser a possibilidade do futuro de aproveitamento de uma fonte de energia ilimitada, a luz solar. Desde então, a ciência não parou mais de investir e evoluir nessa temática.

Atualmente, contam-se com painéis fotovoltaicos de alta capacidade de geração de energia. Esses painéis podem ser isolados (Off-Grid) ou conectados (On-Grid). Os sistemas isolados são aqueles que necessitam de um sistema de armazenamento utilizando baterias, ou seja, não possuem conexão com a rede de distribuição das concessionárias de energia. Já o sistema conectado é aquele vinculado à rede elétrica de distribuição de energia, e nesse caso, os painéis funcionam como um pequeno fornecedor de energia para a rede. Essa já é uma realidade para a geração de energia elétrica limpa e sustentável (Rüther e Salamoni, 2013). "Nesse sentido, a geração distribuída é uma tendência por conta da autonomia dos consumidores de gerar sua própria energia" (David e Sabbadini, 2017, p. 2).

Segundo Di Souza (2016), como sistemas On-Grid utilizam a rede de distribuição para escoamento da energia gerada, esses tipos de sistemas dependem de regulamentação e legislação favorável à sua realização. Neste contexto, o Brasil criou a Resolução Normativa no 482/2012, que define o Sistema de Compensação de Energia Elétrica (ANEEL, 2012). Esta resolução determina que o consumidor brasileiro pode gerar sua própria energia elétrica a partir da utilização de fontes renováveis e fornecer o excedente para a rede de distribuição de sua localidade (ANEEL, 2016).

No que se refere às políticas públicas, o Brasil criou o Programa de Desenvolvimento da Geração Distribuída de Energia Elétrica (ProGD) (Brasil, 2015), que visa a incentivar a geração distribuída de energias renováveis. O programa vem disponibilizando linhas de crédito e financiamentos para a geração distribuída, o que também impulsiona as indústrias de equipamentos, e ainda a formação de mão de obra especializada. Estima-se que, até 2030, o ProGD tenha investido 100 bilhões de reais, com adesão de 2,7 milhões de unidades consumidoras e geração de 48 milhões de megawattshora (MWh) (Brasil, 2015; David e Sabbadini, 2017). Outro programa de incentivo é o Programa para o Desenvolvimento da Energia para Estados e Municípios (PRODEEM), que tem por objetivo contribuir para o desenvolvimento de comunidades não atendidas pelos sistemas convencionais de geração e distribuição de energia. Há ainda o programa da Caixa Econômica Federal (CEF) intitulado Construcard, que viabiliza o financiamento de 
instalações de geração de energias renováveis como a eólica e a solar fotovoltaica (David e Sabbadini, 2017).

Portanto, a geração de energia solar vem se tornando a proposta mais promissora para a geração de energia limpa e sustentável, visto que, a energia que a Terra recebe do sol anualmente é cerca de 10.000 vezes maior do que o consumo mundial. Machado e Miranda (2014) relatam que, no ano 2000, o consumo mundial de energia era de 13 Terawatts (TW) e, estima-se que em 2050 a demanda seja de 30 TW, ainda assim, nota-se que há um excedente de energia solar. 0 maior problema encontrado para a captação de energia solar ainda são os custos elevados de implantação dos sistemas fotovoltaicos. Ainda que os investimentos sejam altos, a Agência Internacional de Energia (IEA, 2018) afirma que o mercado global de energia solar fotovoltaica cresceu de forma significativa, apresentando um aumento de $98 \mathrm{GW}$ da capacidade instalada em 2017. Além disso, os sistemas de energia solar representam possibilidades de levar a eletricidade a comunidades isoladas e aproveitamento em áreas já construídas (Rüther e Salamoni, 2013).

Segundo Barragán-Escandón et al. (2019), a energia solar apresenta grandes vantagens, uma vez que o fornecimento por meio de um sistema fotovoltaico está ligado diretamente com o espaço disponível para a colocação das placas, ou seja, um município pode ser visto como uma usina fotovoltaica.

Silva e Romero (2015) relatam que $80 \%$ da população brasileira está localizada em áreas urbanas, e os índices apontam que nos próximos 30 anos o crescimento populacional continuará concentrado na zona urbana. Embora o Brasil ainda esteja iniciando nesse setor de geração de energia solar fotovoltaica, a implantação desses painéis vem assumindo um papel decisivo na transformação socioambiental (Rüther e Salamoni, 2013). Silva e Romero (2015) também pontuam que foram executadas muitas ações na América Latina a fim de assumir desafios e adversidades urbanas a partir de uma perspectiva de sustentabilidade, o que demanda incluir a dimensão energética nas políticas públicas. Para eles, a gestão urbana sustentável deve ser estudada a partir do nível local.

Dada à importância e a abrangência das temáticas relacionadas à produção e consumo de energia, cabe aqui ressaltar que a Agenda dos Objetivos de Desenvolvimento do Milênio (ODM), proposta pela Organização das Nações Unidas (ONU), em 2000, não trouxe dentre seus oito objetivos, e suas respectivas metas, o compromisso com a geração de energia limpa e sustentável. Jannuzzi e Carlo (2018) citam os ODM como uma abordagem minimalista, não apenas nesse aspecto, mas de maneira geral.

Já em 2015, foram elaborados também pela ONU, os Objetivos de Desenvolvimento Sustentável (ODS), que devem ser implementados em todos os países do mundo até 2030 (ONU, 2015). Para Jannuzzi e Carlo (2018), os ODS são mais abrangentes em suas temáticas e mais minuciosos em suas metas e planos de ação. Os ODS são divididos em dezessete tópicos, e o ODS 7 trata especificamente de "Assegurar o acesso confiável, sustentável, moderno e a preço acessível de energia para todos". Logo, as metas estabelecidas para concretizar esse objetivo visam ao acesso universal a preços acessíveis; o aumento da participação de energias renováveis na matriz energética global; a melhoria da eficiência energética; o reforço da cooperação internacional para investimentos em pesquisas, tecnologias, e energia limpa; e a modernização do fornecimento e das tecnologias para países em desenvolvimento (ONU, 2015).

De acordo com a Empresa Brasileira de Pesquisa Agropecuária (Kunz et al., 2018), o ODS 7 tende a contribuir imensamente na redução das desigualdades sociais e econômicas, e ainda ajuda a reduzir os problemas ambientais. Essa afirmação se deve ao fato de que a geração de energia de maneira limpa e renovável/sustentável reduz a necessidade de geração de energia a base de combustíveis fósseis. Isto é, a disponibilidade de recursos que estão próximos e são renováveis, tais como, luz solar, ventos, marés,

Rev. Bras. Gest. Amb. Sustent., 2020, vol. 7, n. 15, p. 3-19. 
fontes termais e biomassa, reduzem os custos de produção e de acesso, bem como a degradação ambiental, e possibilitam a geração de renda e segurança energética.

Atualmente $19,3 \%$ da energia consumida no planeta é produzida de maneira renovável e, esse tipo de produção gera cerca de 9,8 milhões de empregos. De acordo com Barragán-Escandón et al. (2019), o uso de tecnologias renováveis e a introdução de fontes de energia vão ao encontro da necessidade de conhecimentos diversificados, ou seja, de pessoal com diferentes graus de qualificação. Nesse contexto, a geração de energia fotovoltaica e de biocombustíveis se destaca entre as demais, e nos últimos anos veem recebendo mais investimentos do que as fontes de energia fósseis (Kunz et al., 2018).

Com o propósito de alcançar o ODS 7, tem-se investido bilhões de dólares em geração de energias renováveis. Da mesma forma, o Brasil vem investindo cada vez mais na elaboração de políticas públicas para geração de energias limpas e renováveis (Kunz et al., 2018). Portanto, fica claro a importância de estudos que versem sobre essa temática, com intuito de levar conhecimento aos cidadãos e aprimorar cada vez mais as políticas públicas nacionais.

\section{Metodologia}

Trata-se de uma pesquisa descritiva, quanto aos objetivos, com abordagem quantitativa, cuja a unidade de análise são os campi da UNESP localizados em 24 municípios do Estado de São Paulo, sendo eles Araçatuba, Araraquara, Assis, Bauru, Botucatu, Dracena, Franca, Guaratinguetá, Ilha Solteira, Itapeva, Jaboticabal, Marília, Ourinhos, Presidente Prudente, Registro, Rio Claro, Rosana, São João da Boa Vista, São José do Rio Preto, São José dos Campos, São Paulo, São Vicente, Sorocaba e Tupã (Figura 1).

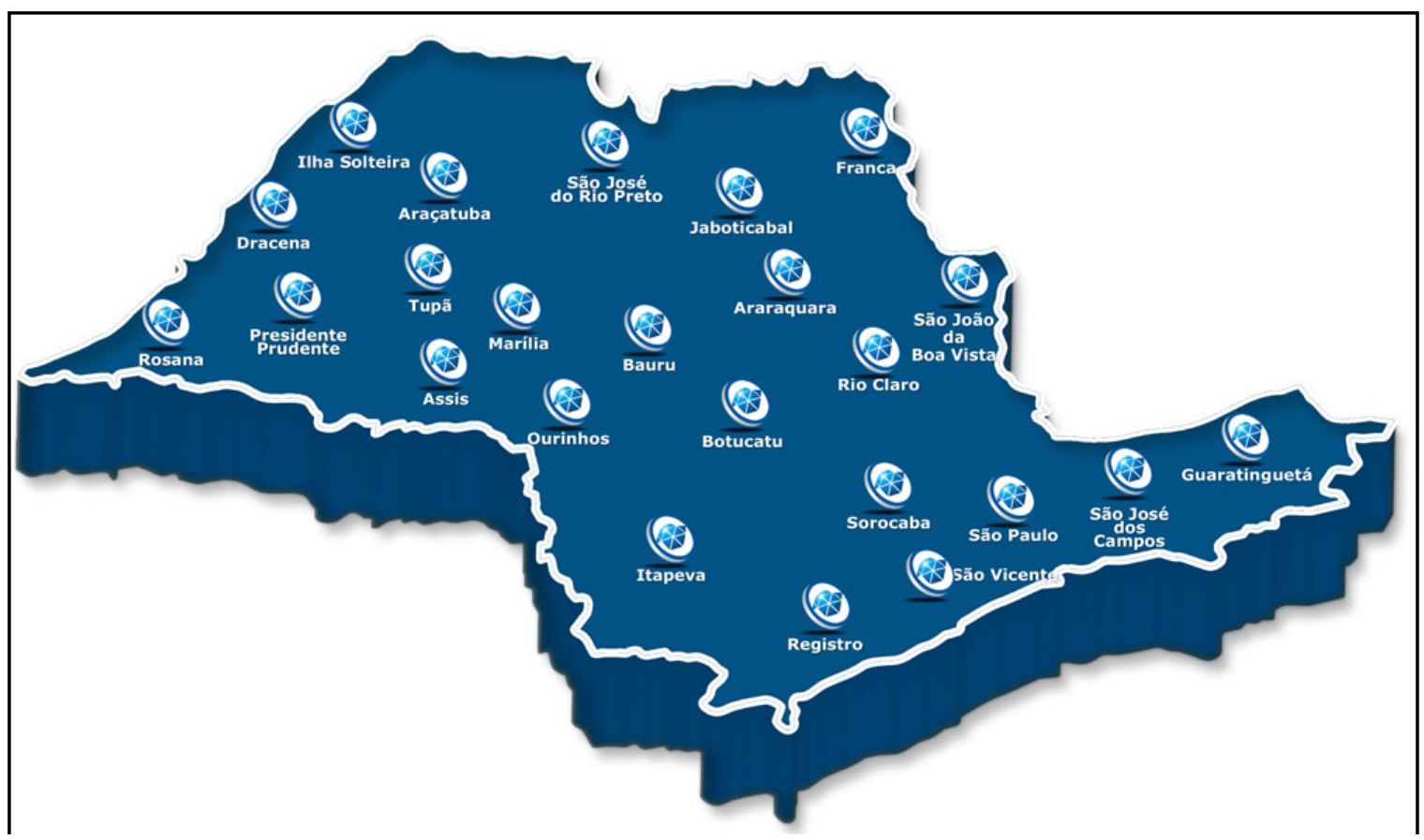

Figura 1. Municípios com instalação de unidades da UNESP. Fonte: Portal UNESP.

Para a realização dessa pesquisa, elaborou-se um banco de dados constituído pela área construída das unidades da UNESP, quantidade diária de energia gerada esperada, componente de radiação diário que atinge diretamente a superfície, componente 
de radiação diário espalhado pela atmosfera, radiação difusa, temperatura $\left({ }^{\circ} \mathrm{C}\right)$ e altitude de cada munícipio onde se encontram localizados os campi, a fim de mensurar o potencial de captação de energia solar fotovoltaica e o custo de implantação do sistema.

As áreas construídas dos campi foram obtidas por meio de consulta ao Anuário Estatístico (UNESP, 2016). Para efetuar a análise, nos municípios que possuem mais de uma unidade foram somadas as suas respectivas áreas. Os dados referentes à radiação solar e a altitude foram extraídos dos Atlas Solares Globais.

Após o levantamento dos dados, foi conduzida uma análise descritiva para examinar as particularidades dos dados e a forma da distribuição das variáveis. Esse tipo de análise é de extrema importância, pois serve para organizar, resumir e permite descrever questões importantes de um conjunto de características observadas ou comparar características entre conjuntos.

Para compreender o inter-relacionamento entre os municípios do estado de São Paulo onde se encontram localizados os campi da UNESP, utilizou-se de uma técnica de análise de clusters ou agrupamentos, que tem por objetivo agregar elementos com base em características similares, resultando em elevada homogeneidade interna (dentro dos agrupamentos) e heterogeneidade externa (entre agrupamentos) (Hair Jr. et al., 2009). Esse método tem sido difundido por diversos autores para determinar ambientes climatologicamente homogêneos (Keller Filho et al., 2005; Fernandes et al., 2012).

Para o agrupamento, foi aplicado o procedimento hierárquico aglomerativo. Um dos métodos mais utilizados na análise de clusters é o Método de Ward, que tende a combinar agrupamentos a partir da minimização da variância interna dos conglomerados, onde se inicia com cada objeto em um grupo próprio. Posteriormente, dois grupos são combinados para formar um novo grupo agregado e assim sucessivamente, formando grupos cada vez maiores (Hair Jr. et al., 2009). O Método de Ward baseia-se na construção de uma matriz de semelhança ou diferenças, cujo objetivo "é minimizar o quadrado da distância euclidiana às médias dos conglomerados" (Fechine e Galvíncio, 2008, p. 79-80).

Além disso, consiste na representação desse processo por meio de um dendrograma ou gráfico em forma de árvore. 0 dendrograma expressa, no sentido da reta horizontal, a distância que cada cluster foi formado e, no sentido da reta vertical, a que distância cada objeto está em relação ao outro.

Como os dados coletados são mensurados em unidades distintas, seus valores foram padronizados por meio de $\mathrm{Z}$ escores, uma vez que a padronização de variáveis possibilita alcançar resultados mais confiáveis para uma análise com melhor qualidade e precisão (Hair Jr. et al., 2009).

Utilizou-se, então, a técnica multivariada de análise de variância (ANOVA), visando a identificar diferenças significativas entre os clusters formados. Essa técnica permite analisar mais de duas populações (k populações) independentes simultaneamente por meio de $\mathrm{k}$ amostras (também independentes).

A ANOVA faz uma análise da variação total de grupos de dados de duas formas diferentes: 1) Variação dentro dos grupos (variância residual ou variância dos erros): avalia a discrepância dos dados em relação às médias dos grupos que pertencem; e, 2) Variação entre grupos (variância do fator ou do tratamento): avalia a discrepância existente entre as médias dos vários grupos. Assim, se a variância dentro dos grupos for significativamente inferior à variância entre grupos, a hipótese da igualdade das médias não é verificada.

Essa análise comporta uma razão (ou estatística) $\mathrm{F}$ que é calculada pela razão entre as duas variações, ou seja, o numerador indica a variação entre os grupos ou tratamentos em função dos graus de liberdade $(\mathrm{k}-1)$, e o denominador indica a variação dentro dos grupos ou tratamentos em função dos graus de liberdade (n - k)), onde k é o número de populações em estudo, e n é a soma dos tamanhos das $\mathrm{k}$ amostras extraídas de tais populações. Assim, a estatística do teste tem distribuição F de Snedecor com 
parâmetros (k - 1) e (n - k) e, para um nível de significância $\alpha$, se a estatística $\mathrm{F}$ calculada a partir dos valores amostrais for maior do que a estatística $\mathrm{F}$ teórica, ou equivalentemente, se o valor_p (probabilidade de significância do teste obtida a partir dos dados) for menor ou igual a $\alpha$, rejeita-se a hipótese Ho de igualdade entre as $\mathrm{k}$ populações (Martins e Domingues, 2017).

Finalmente, para localizar as diferenças entre as amostras, aplicou-se o teste de Tukey, que testa as amostras duas a duas para ver quais pares de grupos, de fato, possuem diferenças significativas.

Neste trabalho, para o tratamento estatístico, foram utilizados os softwares BioEstat 5.3 e SPSS 24.0, e foi considerado um nível de significância $\alpha$ de $5 \%$ para os testes de hipóteses realizados.

\section{Resultados e discussão}

Primeiramente, foram calculadas as medidas descritivas para os dados das variáveis consideradas, conforme Tabela 1.

Tabela 1. Medidas descritivas das variáveis analisadas.

\begin{tabular}{|c|c|c|c|c|c|}
\hline Variáveis & $\begin{array}{c}\text { Média } \\
\text { Aritmética }\end{array}$ & $\begin{array}{l}\text { Desvio } \\
\text { Padrão } \\
\end{array}$ & $\begin{array}{c}\text { Valor } \\
\text { Mínimo }\end{array}$ & $\begin{array}{c}\text { Valor } \\
\text { Máximo }\end{array}$ & $\begin{array}{l}\mathrm{CV}^{*} \\
(\%) \\
\end{array}$ \\
\hline $\begin{array}{l}\text { Quantidade de energia } \\
\text { (kWh/kWp por dia) }\end{array}$ & 4,28 & 0,30 & 3,38 & 4,52 & 7 \\
\hline $\begin{array}{l}\text { Radiação na superfície } \\
\text { (kWh } / \mathrm{m}^{2} \text { por dia) }\end{array}$ & 4,69 & 0,65 & 2,94 & 5,47 & 14 \\
\hline $\begin{array}{c}\text { Radiação na atmosfera } \\
\left(\mathrm{kWh} / \mathrm{m}^{2} \text { por dia) }\right.\end{array}$ & 1,99 & 0,07 & 2,11 & 1,84 & 3 \\
\hline $\begin{array}{c}\text { Radiação difusa } \\
\left(\mathrm{kWh} / \mathrm{m}^{2} \text { por dia) }\right.\end{array}$ & 5,09 & 0,38 & 4,07 & 5,50 & 7,5 \\
\hline $\begin{array}{l}\text { Radiação difusa nos painéis } \\
\text { (kWh } / \mathrm{m}^{2} \text { por dia) }\end{array}$ & 5,46 & 0,42 & 4,30 & 5,88 & 7,6 \\
\hline $\begin{array}{c}\text { Temperatura } \\
\left({ }^{\circ} \mathrm{C}\right)\end{array}$ & 21,29 & 1,78 & 18,30 & 24,50 & 8,4 \\
\hline $\begin{array}{l}\text { Altitude } \\
\text { (m) }\end{array}$ & 542,54 & 231,58 & 7 & 1028 & 43 \\
\hline
\end{tabular}

*O coeficiente de variação mede a dispersão relativa dos dados em relação à média, ou seja, é a razão entre o desvio-padrão e a média aritmética. Se for menor que 0,15 ou $15 \%$, existe pouca dispersão dos dados.

Por meio do coeficiente de variação (CV) e dos valores mínimos e máximos obtidos, verifica-se que a quantidade de energia, as radiações e a temperatura dos municípios são homogêneas, ou seja, têm pouca variabilidade. Apenas a altitude apresenta uma elevada dispersão de um município para o outro (Martins e Domingues, 2017).

Posteriormente, realizou-se uma análise de clusters para identificar em quais épocas do ano há maior incidência de luz solar nos municípios em estudo. Apresenta-se na Figura 2 o dendrograma formado, onde o eixo horizontal indica o nível de similaridade (segundo uma distância euclidiana quadrática) e, o eixo vertical, os meses em que possuem incidências semelhantes.

A análise de agrupamento aplicada aos dados de quantidade de incidência solar e altitude dos 24 municípios onde se encontram localizados os campi da UNESP, possibilitou 
distinguir três clusters homogêneos, com corte na distância 5. Nota-se que os agrupamentos formados pelos meses são influenciados pelas estações do ano, uma vez que os meses de maio, junho e julho são de transição do outono para o inverno e, sendo assim, apresentam uma menor incidência de radiação. Da mesma forma, os meses de novembro a fevereiro são de transição da primavera para o verão, onde há uma elevada precipitação, chamada de chuvas de verão (Pereira et al., 2006).

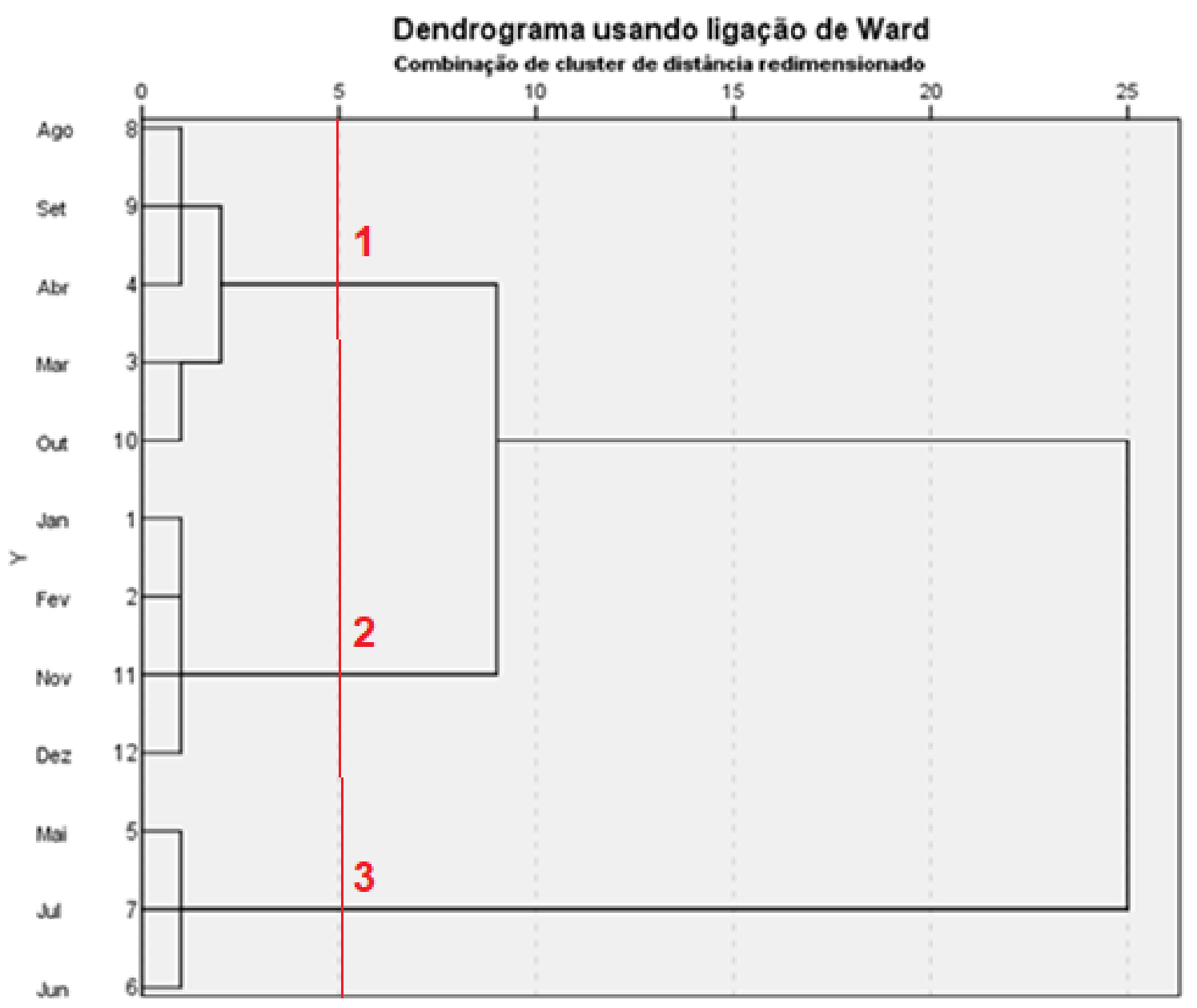

Figura 2. Dendrograma obtido pelo Método de Ward, com dados de médias de incidência solar nos municípios analisados.

A fim de observar os municípios que apresentam características similares baseadas nas variáveis de incidência solar e altitude, utilizadas neste trabalho, aplicou-se novamente a análise de clusters. Essa análise é representada pelo dendograma da Figura 3, onde o eixo horizontal indica o nível de similaridade (segundo uma distância euclidiana quadrática) e, o eixo vertical, os municípios em estudo. 


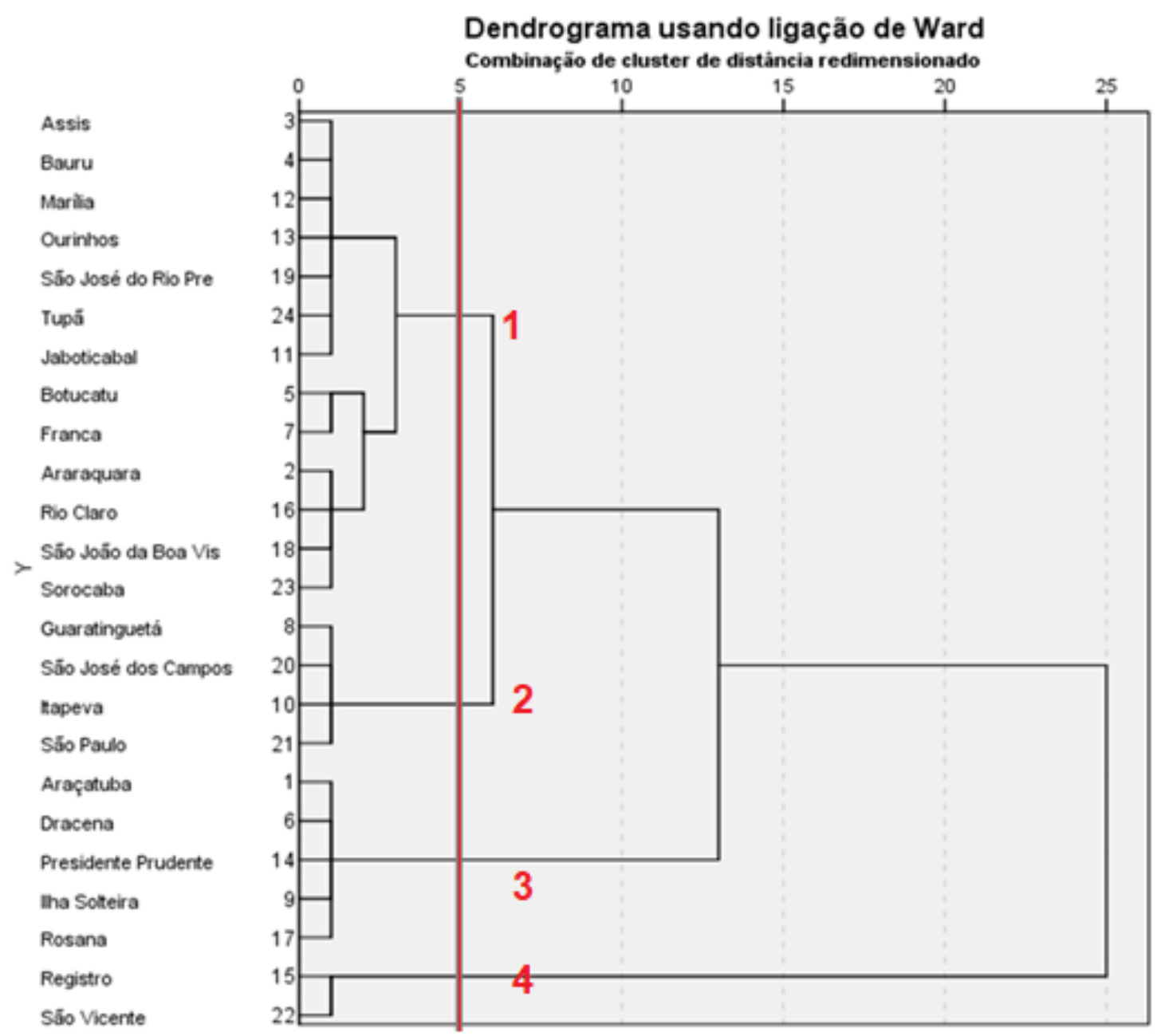

Figura 3. Dendrograma obtido pelo Método de Ward, com dados dos 24 municípios do Estado de São Paulo.

Devido ao fato de os municípios paulistas apresentarem uma variação ínfima na quantidade de incidência solar, optou-se por fazer um corte na distância 5, para que as formações dos grupos ocorressem de forma mais criteriosa.

Assim, foram identificados quatro clusters de municípios com características de incidência solar e altitude similares. No cluster 1 , as maiores similaridades são encontradas entre Araçatuba, Dracena, Presidente Prudente, Ilha Solteira e Rosana, o que corresponde a $21 \%$ dos municípios, e isso já era esperado por possuírem temperaturas mais elevadas. 0 cluster 2 é formado pelos munícipios de Assis, Bauru, Marília, Ourinhos, São José do Rio Preto, Tupã, Jaboticabal, Botucatu, Franca, Araraquara, Rio Claro, São João da Boa Vista e Sorocaba (54\%) por apresentarem altitudes maiores que os outros municípios em estudo. No cluster 3 encontram-se Guaratinguetá, São José dos Campos, Itapeva e São Paulo (17\%), apresentando temperaturas mais amenas. Por fim, o cluster 4 é formado pelos Municípios de Registro e São Vicente (8\%), pois se encontram próximos ao nível do mar.

Como os índices de incidência solar e altitude dos municípios são semelhantes, aplicou-se a ANOVA (vide Tabela 2), a fim de confirmar a existência de dessemelhança 
entre os grupos de municípios que contam com os campi da UNESP. Para um nível de 5\%, concluiu-se então a existência de diferença significativa dos índices médios de incidência solar e altitude entre os quatro clusters de municípios formados, evidenciando uma homogeneidade interna para tais agrupamentos.

Tabela 2. ANOVA dos quatro clusters formados na Figura 3.

\begin{tabular}{|c|c|c|c|c|c|c|}
\hline \multicolumn{2}{|l|}{ ANOVA } & $\begin{array}{c}\text { Soma dos } \\
\text { Quadrados }\end{array}$ & $\begin{array}{c}\text { Grau de } \\
\text { Liberdade }\end{array}$ & $\begin{array}{c}\text { Quadrado } \\
\text { Médio }\end{array}$ & $\mathbf{F}$ & valor_p \\
\hline \multirow{3}{*}{$\begin{array}{c}\text { Quantidade de } \\
\text { energia }\end{array}$} & $\begin{array}{c}\text { Entre } \\
\text { clusters }\end{array}$ & 1,99 & 3 & 0,66 & 118,45 & $0,000^{*}$ \\
\hline & $\begin{array}{c}\text { Nos } \\
\text { clusters }\end{array}$ & 0,11 & 20 & 0,01 & & \\
\hline & Total & 2,10 & 23 & & & \\
\hline \multirow{3}{*}{ Radiação difusa } & $\begin{array}{c}\text { Entre } \\
\text { clusters }\end{array}$ & 2,90 & 3 & 0,97 & 87,71 & $0,000^{*}$ \\
\hline & $\begin{array}{c}\text { Nos } \\
\text { clusters }\end{array}$ & 0,22 & 20 & 0,01 & & \\
\hline & Total & 3,12 & 23 & & & \\
\hline \multirow{3}{*}{$\begin{array}{l}\text { Radiação na } \\
\text { superfície }\end{array}$} & $\begin{array}{c}\text { Entre } \\
\text { clusters }\end{array}$ & 9,20 & 3 & 3,07 & 98,51 & $0,000^{*}$ \\
\hline & $\begin{array}{c}\text { Nos } \\
\text { clusters }\end{array}$ & 0,62 & 20 & 0,03 & & \\
\hline & Total & 9,83 & 23 & & & \\
\hline \multirow{3}{*}{$\begin{array}{l}\text { Radiação na } \\
\text { atmosfera }\end{array}$} & $\begin{array}{c}\text { Entre } \\
\text { clusters }\end{array}$ & 0,07 & 3 & 0,03 & 20,48 & $0,000^{*}$ \\
\hline & $\begin{array}{c}\text { Nos } \\
\text { clusters }\end{array}$ & 0,02 & 20 & 0,001 & & \\
\hline & Total & 0,10 & 23 & & & \\
\hline \multirow{3}{*}{$\begin{array}{c}\text { Radiação difusa } \\
\text { nos painéis }\end{array}$} & $\begin{array}{c}\text { Entre } \\
\text { clusters }\end{array}$ & 3,44 & 3 & 1,15 & 68,53 & $0,000^{*}$ \\
\hline & $\begin{array}{c}\text { Nos } \\
\text { clusters }\end{array}$ & 0,34 & 20 & 0,02 & & \\
\hline & Total & 3,78 & 23 & & & \\
\hline \multirow{3}{*}{ Temperatura } & $\begin{array}{c}\text { Entre } \\
\text { clusters }\end{array}$ & 57,18 & 3 & 19,06 & 23,93 & $0,000^{*}$ \\
\hline & $\begin{array}{c}\text { Nos } \\
\text { clusters }\end{array}$ & 15,93 & 20 & 0,80 & & \\
\hline & Total & 73,11 & 23 & & & \\
\hline \multirow{3}{*}{ Altitude } & $\begin{array}{c}\text { Entre } \\
\text { clusters }\end{array}$ & 908521,03 & 3 & 302840,343 & 18,64 & $0,000^{*}$ \\
\hline & $\begin{array}{c}\text { Nos } \\
\text { clusters }\end{array}$ & 324978,93 & 20 & 16248,947 & & \\
\hline & Total & 1233499,96 & 23 & & & \\
\hline
\end{tabular}

*Significativo para um nível de significância de 5\%.

A fim de comparar os clusters entre si, e verificar quais são significativamente iguais (ou diferentes), foi aplicado a teste de Tukey, com resultados apresentados na Tabela 3. Pode-se observar que, para todas as variáveis em estudo, há diferenças significativas entre os pares de clusters, ao nível de significância de 5\%. 
Tabela 3. Comparações múltiplas (Teste de Tukey).

\begin{tabular}{|c|c|c|c|c|c|}
\hline Variável dependente & $\begin{array}{l}\text { (I) Ward } \\
\text { Method }\end{array}$ & $\begin{array}{l}\text { (J) Ward } \\
\text { Method }\end{array}$ & $\begin{array}{c}\text { Diferença } \\
\text { média } \\
(\mathrm{I}-\mathrm{J}) \\
\end{array}$ & $\begin{array}{l}\text { Erro } \\
\text { Padrão }\end{array}$ & valor_p \\
\hline \multirow{6}{*}{ Quantidade de energia } & \multirow{3}{*}{1} & 2 & 0,05898 & 0,03937 & 0,460 \\
\hline & & 3 & 0,35410 & 0,05019 & $0,000^{*}$ \\
\hline & & 4 & 1,05060 & 0,0626 & $0,000^{*}$ \\
\hline & \multirow{2}{*}{2} & 3 & 0,29512 & 0,04278 & $0,000^{*}$ \\
\hline & & 4 & 0,99162 & 0,05683 & $0,000^{*}$ \\
\hline & 3 & 4 & 0,69650 & 0,0648 & $0,000^{*}$ \\
\hline \multirow{6}{*}{ Radiação difusa } & \multirow{3}{*}{1} & 2 & 0,17495 & 0,05522 & $0,020^{*}$ \\
\hline & & 3 & 0,56855 & 0,07039 & $0,000^{*}$ \\
\hline & & 4 & 1,28880 & 0,08779 & $0,000^{*}$ \\
\hline & \multirow{2}{*}{2} & 3 & 0,39360 & 0,06 & $0,000^{*}$ \\
\hline & & 4 & 1,11385 & 0,0797 & $0,000^{*}$ \\
\hline & 3 & 4 & 0,72025 & 0,09087 & $0,000^{*}$ \\
\hline \multirow{6}{*}{ Radiação na superfície } & \multirow{3}{*}{1} & 2 & 0,40252 & 0,09286 & $0,000^{*}$ \\
\hline & & 3 & 1,13360 & 0,11837 & $0,000^{*}$ \\
\hline & & 4 & 2,29660 & 0,14764 & $0,000^{*}$ \\
\hline & \multirow{2}{*}{2} & 3 & 0,73108 & 0,1009 & $0,000^{*}$ \\
\hline & & 4 & 1,89408 & 0,13403 & $0,000^{*}$ \\
\hline & 3 & 4 & 1,16300 & 0,15282 & $0,000^{*}$ \\
\hline \multirow{6}{*}{ Radiação na atmosfera } & \multirow{3}{*}{1} & 2 & $-0,11462$ & 0,01813 & $0,000^{*}$ \\
\hline & & 3 & $-0,14100$ & 0,02311 & $0,000^{*}$ \\
\hline & & 4 & $-0,18200$ & 0,02883 & $0,000^{*}$ \\
\hline & \multirow{2}{*}{2} & 3 & $-0,02638$ & 0,0197 & 0,550 \\
\hline & & 4 & $-0,06738$ & 0,02617 & 0,080 \\
\hline & 3 & 4 & $-0,041$ & 0,02984 & 0,530 \\
\hline \multirow{6}{*}{$\begin{array}{l}\text { Radiação difusa nos } \\
\text { painéis }\end{array}$} & \multirow{3}{*}{1} & 2 & 0,16115 & 0,0681 & 0,120 \\
\hline & & 3 & 0,52400 & 0,08681 & $0,000^{*}$ \\
\hline & & 4 & 1,42750 & 0,10827 & $0,000^{*}$ \\
\hline & \multirow{2}{*}{2} & 3 & 0,36285 & 0,07399 & $0,000^{*}$ \\
\hline & & 4 & 1,26635 & 0,09829 & $0,000^{*}$ \\
\hline & 3 & 4 & 0,90350 & 0,11207 & $0,000^{*}$ \\
\hline \multirow{6}{*}{ Temperatura } & \multirow{3}{*}{1} & 2 & 2,66308 & 0,46961 & $0,000^{*}$ \\
\hline & & 3 & 5,01500 & 0,59864 & $0,000^{*}$ \\
\hline & & 4 & 2,09000 & 0,74664 & $0,050^{*}$ \\
\hline & \multirow{2}{*}{2} & 3 & 2,35192 & 0,51025 & $0,000^{*}$ \\
\hline & & 4 & $-0,57308$ & 0,67783 & 0,830 \\
\hline & 3 & 4 & $-292,500$ & 0,77284 & $0,010^{*}$ \\
\hline \multirow{6}{*}{ Altitude } & \multirow{3}{*}{1} & 2 & 276,86154 & 67,07986 & $0,003^{*}$ \\
\hline & & 3 & 292,40000 & 85,51039 & $0,013^{*}$ \\
\hline & & 4 & 357,10000 & 106,65019 & $0,016^{*}$ \\
\hline & \multirow{2}{*}{2} & 3 & -1553846 & 72,88454 & 0,996 \\
\hline & & 4 & 633,96154 & 96,82145 & $0,000^{*}$ \\
\hline & 3 & 4 & 649,50000 & 110,39343 & $0,000^{*}$ \\
\hline
\end{tabular}

*Significativo para um nível de significância de 5\%.

Assim, os clusters formados a partir das características similares apresentadas na Figura 3, podem servir de base para determinar a captação de energia solar e geração de energia elétrica por células fotovoltaicas. Para tanto, foi selecionado de cada cluster 0 
campus com a menor unidade de área construída, a fim de aferir o custo de implantação e o potencial de captação de energia de um sistema por painéis fotovoltaicos nessas unidades da UNESP.

Como mostra a Tabela 4 a seguir, os campi com menores áreas, dentro de cada cluster, são: Rosana (cluster 1), Ourinhos (cluster 2), Itapeva (cluster 3) e São Vicente (cluster 4), respectivamente.

Tabela 4. Campus com menor área construída dentro de cada cluster formado.

\begin{tabular}{c|c|c}
\hline Cluster & Campus & Área construída (m $\mathbf{~}$ \\
\hline 1 & Rosana & 4525 \\
\hline 2 & Ourinhos & 1850 \\
\hline 3 & Itapeva & 4831 \\
\hline 4 & São Vicente & 2473 \\
\hline
\end{tabular}

Para o estudo foram selecionados seis modelos de placas fotovoltaicas com os seguintes atributos: modelo, dimensões, peso, potência, eficiência, tipo de célula e valor das placas, conforme Tabela 5 .

Tabela 5. Características e valor das placas (Junho de 2019).

\begin{tabular}{|c|c|c|c|c|c|c|c|c|c|}
\hline $\begin{array}{l}\frac{0}{0} \\
\frac{0}{2}\end{array}$ & 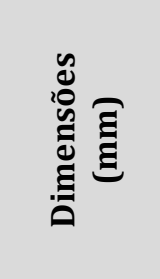 & 导 & 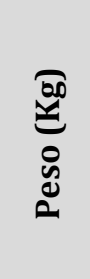 & 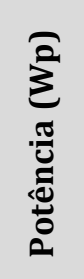 & 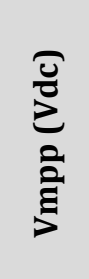 & 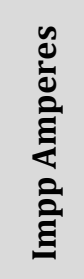 & : & 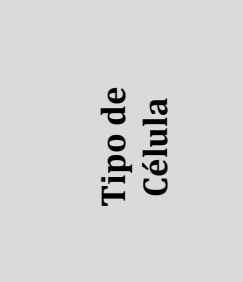 & $\begin{array}{l}\text { ỹ } \\
\frac{\pi}{0} \\
\frac{\pi}{2} \\
\frac{\pi}{0} \\
\frac{\pi}{0} \\
\frac{0}{0}\end{array}$ \\
\hline KS20T & $\begin{array}{c}520 \times 352 \\
\times 22\end{array}$ & 0,183 & 2,4 & 20 & 17,4 & 1,16 & $11 \%$ & $\begin{array}{l}\text { Silíco } \\
\text { Policristalino }\end{array}$ & $\mathrm{R} \$ 222,27$ \\
\hline KS45T & $\begin{array}{c}1028 \times \\
343 \times 36\end{array}$ & 0,353 & 4,55 & 45 & 17,4 & 2,55 & $12,80 \%$ & $\begin{array}{l}\text { Silíco } \\
\text { Policristalino }\end{array}$ & $\mathrm{R} \$ 296,67$ \\
\hline $\begin{array}{l}\text { REM36-6- } \\
\text { 150P }\end{array}$ & $\begin{array}{c}1480 \times \\
680 \times 30\end{array}$ & 10,064 & 11 & 150 & 18,3 & 8,81 & $15,15 \%$ & $\begin{array}{l}\text { Silíco } \\
\text { Policristalino }\end{array}$ & $\mathrm{R} \$ 389,67$ \\
\hline ТР672P & $\begin{array}{c}1650 \times \\
992 \times 35\end{array}$ & 16,368 & 19 & 270 & 31,3 & 9,09 & $16,50 \%$ & $\begin{array}{l}\text { Silíco } \\
\text { Policristalino }\end{array}$ & $\mathrm{R} \$ 519,87$ \\
\hline $\begin{array}{l}\text { CS6K300 } \\
\text { MS }\end{array}$ & $\begin{array}{c}1650 \times \\
992 \times 40\end{array}$ & 16,368 & 18,2 & 300 & 32,5 & 9,24 & $18,33 \%$ & $\begin{array}{l}\text { Silíco } \\
\text { Monocristalino }\end{array}$ & $\mathrm{R} \$ 631,47$ \\
\hline $\begin{array}{l}\text { CS6U- } \\
325 \mathrm{P}\end{array}$ & $\begin{array}{c}1960 \times \\
992 \times 40\end{array}$ & $\begin{array}{c}194,43 \\
2\end{array}$ & 22,4 & 325 & 37 & 9,34 & $16,72 \%$ & $\begin{array}{l}\text { Silíco } \\
\text { Policristalino }\end{array}$ & $\mathrm{R} \$ 650,07$ \\
\hline
\end{tabular}

Fonte: MINHA CASA SOLAR - L\&F Comércio, Projetos e Consultoria LTDA.

Assim, os cálculos foram feitos a partir da equação (1), proposta por Lopez (2012, p. 94), dada por:

$$
P_{g}=\eta \cdot A \cdot R_{s}(t)
$$

onde: $\eta$ : rendimento do sistema; $A$ : área do painel solar e $R_{s}(t)$ : radiação solar incidente em função do tempo. 
Ressalta-se que foi desconsiderado o rendimento da potência instalada, uma vez que essa potência considera todo o restante da instalação, principalmente características e quantidades de baterias. Da mesma forma, como não se possui o mapa de insolação de cada município, considerou-se para os cálculos uma média de seis horas de radiação sem variação.

A Tabela 6 contém os dados relativos à quantidade de placas necessárias para a instalação dos painéis em toda área coberta de cada campus selecionado, o potencial de geração e o custo.

Tabela 6. Análise da viabilidade de instalação das placas para cada campus selecionado.

\begin{tabular}{|c|c|c|c|c|}
\hline Modelo & $\begin{array}{c}\text { Quantidade de } \\
\text { Placas }\end{array}$ & $\begin{array}{l}\text { Potência e Energia } \\
\text { gerada (kWh/dia) }\end{array}$ & $\begin{array}{l}\text { Potência e energia } \\
\text { gerada (MWh/dia) }\end{array}$ & \begin{tabular}{|c|}
$\begin{array}{c}\text { Custo Total } \\
\text { (R\$) }\end{array}$ \\
\end{tabular} \\
\hline \multicolumn{5}{|c|}{ Rosana } \\
\hline KS20T & 24726 & 15909,61 & 15,91 & $5.495 .848,02$ \\
\hline KS45T & 12835 & 18513,01 & 18,51 & $3.807 .759,45$ \\
\hline REM36-6-150P & 4497 & 21911,88 & 21,91 & $1.752 .345,99$ \\
\hline TP672P & 2765 & 23864,42 & 23,86 & $1.437 .440,55$ \\
\hline CS6K300MS & 2765 & 26511,20 & 26,51 & $1.746 .014,55$ \\
\hline CS6U-325P & 2327 & 24182,61 & 24,18 & $1.512 .712,89$ \\
\hline \multicolumn{5}{|c|}{ Ourinhos } \\
\hline KS20T & 10107 & 5857,14 & 5,86 & $2.246 .482,89$ \\
\hline KS45T & 5246 & 6815,58 & 6,82 & $1.556 .330,82$ \\
\hline REM36-6-150P & 1838 & 8066,88 & 8,07 & $716.213,46$ \\
\hline TP672P & 1130 & 8785,71 & 8,79 & $587.453,10$ \\
\hline CS6K300MS & 1130 & 9760,12 & 9,76 & $713.561,10$ \\
\hline CS6U-325P & 951 & 8902,85 & 8,90 & $618.216,57$ \\
\hline \multicolumn{5}{|c|}{ Itapeva } \\
\hline KS20T & 26393 & 13732,7 & 13,73 & 5.866.372,11 \\
\hline KS45T & 13700 & 15979,87 & 15,98 & $4.064 .379,00$ \\
\hline REM36-6-150P & 4800 & 18913,67 & 18,91 & $1.870 .416,00$ \\
\hline TP672P & 2951 & 20599,05 & 20,60 & $1.534 .136,37$ \\
\hline CS6K300MS & 2951 & 22883,67 & 22,88 & $1.863 .467,97$ \\
\hline CS6U-325P & 2484 & 20873,7 & 20,87 & $1.614 .773,88$ \\
\hline \multicolumn{5}{|c|}{ São Vicente } \\
\hline KS20T & 13510 & 4968,36 & 4,97 & $3.002 .867,70$ \\
\hline KS45T & 7013 & 5781,36 & 5,78 & $2.080 .546,71$ \\
\hline REM36-6-150P & 2457 & 6842,78 & 6,84 & $957.419,19$ \\
\hline TP672P & 1510 & 7452,53 & 7,45 & $785.003,70$ \\
\hline CS6K300MS & 1510 & 8279,09 & 8,28 & $953.519,70$ \\
\hline CS6U-325P & 1271 & 7551,9 & 7,55 & $826.238,97$ \\
\hline
\end{tabular}

Por meio dos cálculos, observa-se que a placa CS6K300MS possui um desempenho de captação solar mais eficiente, não requer uma quantidade muito grande de placas, e o custo é mediano em relação às demais. Em seguida, destaca-se a placa CS6U-325P que, apresenta uma potência um pouco inferior (9\%) a CS6K300MS, mas requer menor quantidade de placas (por ser composta por outro material e ter maior dimensão) e possui um custo de implantação 13\% menor que o da CS6K300MS. Por fim, destaca-se a placa TP672P, que necessita do mesmo número de placas da CS6K300MS, no entanto, apresenta o terceiro melhor desempenho em potência e energia gerada, e tem um custo aproximadamente $17 \%$ menor que o da CS6K300MS, e cerca de $5 \%$ menor que o da 
CS6U-325P. Aparentemente, as demais placas não parecem ter um custo-benefício que as favoreça, conforme a condução do presente estudo.

Ainda que os cálculos apresentados tenham sido feitos para o campus com menor área construída, dentro de cada cluster, deduz-se que a análise realizada na Tabela 6 seja similar para os outros campi pertencentes ao mesmo cluster, dadas as características de incidência solar e altitude que agruparam as unidades da UNESP (Figura 3 e Tabela 2). Ou seja, pode-se prever um planejamento e resultados análogos (mesmos percentuais), em termos de custo e de geração de energia, para os outros campi que compõem os clusters formados.

\section{Conclusão}

Atualmente, muitas pesquisas vêm sendo desenvolvidas acerca da importância da sustentabilidade. Uma das questões fundamentais para o desenvolvimento sustentável encontra-se na busca de inovação e aproveitamento de recursos energéticos naturais (Pereira et al., 2006; Reis, 2015).

Nos últimos anos, a conversão de energia solar em energia elétrica tem ganhado destaque no mundo todo, uma vez que esses sistemas possibilitam distribuir, na rede do sistema elétrico, a energia gerada excedente ao próprio consumo (Nascimento et al., 2015; Strangueto, 2016).

No entanto, a aquisição e instalação de um sistema fotovoltaico, quer seja On-Grid (conectado à rede) ou Off-Grid (sem conexão ou isolado), representam um alto investimento inicial. Ademais, os sistemas isolados necessitam de baterias estacionárias, uma vez que não há geração de energia durante a noite (Nascimento et al., 2015; Strangueto, 2016).

Portanto, é necessário que um planejamento minucioso seja realizado para a aquisição e instalação do referido sistema, de forma que seja garantida a viabilidade do empreendimento com menor custo.

Neste sentido, o presente trabalho apresenta uma metodologia que viabiliza esse planejamento, deduzindo resultados e destacando pontos positivos e negativos ao empreendimento, dando condições à instituição de direcionar ações estratégicas para a implantação de um sistema fotovoltaico. Além disso, a metodologia proposta tem potencial para ser considerada ou expandida a empreendimentos similares.

Por fim, ressalta-se que um estudo complementar ao apresentado pode ser conduzido considerando outras variáveis (características locais), tais como: disponibilidade da área do telhado, características estruturais do telhado (formato, tipo, inclinação, orientação) e dados sobre sombreamento com relação ao movimento do sol sobre a superfície em estudo (ângulo solar de altitude, ângulo azimutal, ângulo horário, ângulo zenital). No entanto, esses dados deverão ser coletados por equipamentos no local e por certo período, para resultados mais precisos.

\section{Conflito de interesses}

Os autores declaram não haver conflito de interesses.

\section{Referências}

ANEEL - Agência Nacional de Energia Elétrica. Micro e minigeração distribuída: sistema de compensação de energia elétrica. Brasília: ANEEL, 2016. (Cadernos temáticos ANEEL). Disponível em: <http://www.aneel.gov.br/documents/656877/14913578/Caderno+tematico+Micro+e+ Minigeração+Distribuida+-+2+edicao/716e8bb2-83b8-48e9-b4c8-a66d7f655161>. Acesso em: 16 fev. 2019.

Rev. Bras. Gest. Amb. Sustent., 2020, vol. 7, n. 15, p. 3-19. 
ANEEL - Agência Nacional de Energia Elétrica. Resolução Normativa no 482, de 17 de abril de 2012. Estabelece as condições gerais para o acesso de microgeração e minigeração distribuída aos sistemas de distribuição de energia elétrica, o sistema de compensação de energia elétrica, e dá outras providências. Disponível em: <http://www2.aneel.gov.br/cedoc/ren2012482.pdf>. Acesso em: 16 fev. 2019.

Barragán-Escandón, E.; Zalamea, E. ; Terrados, J.; Vanegas, P. Factores que influyen en la selección de energías renovables en la ciudad. EURE - Revista Latinoamericana de Estudios Urbano Regionales, v. 45, n. 134, p. 259-277, 2019. https://doi.org/10.4067/S0250-71612019000100259

Borges, F. Q.; Zouain, D. M. A matriz elétrica no Estado do Pará e seu posicionamento na promoção do desenvolvimento sustentável. Planejamento e Políticas Públicas, v. 2, n. 35, p. 187-221, 2011.

Brasil. Ministério de Minas e Energia. ProGD - Programa de Desenvolvimento da Geração Distribuída de Energia Elétrica: ações de estímulo à geração distribuída, com base em fontes renováveis. Brasília: MME, 2015. Disponível em: <http://www.mme.gov.br/ documents/10584/3013891/15.12.2015+Apresentação+ProGD/bee12bc8-e635-42f2-b66cfa5cb507fd06>. Acesso em: 16 jun. 2019.

David, T. M.; Sabbadini, F. S. Políticas públicas e eficiência energética em energia solar: uma análise comparativa entre Brasil e Alemanha. Anais do Simpósio de Excelência em Gestão e Tecnologia, Rio de Janeiro, Universidade Federal do Rio de Janeiro, 2017.

Di Souza, R. Energia solar: sistemas de energia solar fotovoltaicos. Ribeirão Preto: BlueSol, 2016.

Farias, L. M.; Sellitto, M. A. Uso da energia ao longo da história: evolução e perspectivas futuras. Revista Liberato, v. 12, n. 17, p. 01-106, 2011. https://doi.org/10.31514/rliberato. 2011v12n17.p07

Fechine, J. A. L.; Galvíncio, J. D. Análise das precipitações mensais da Bacia Hidrográfica do Rio Brígida no semi-árido pernambucano, usando o Método de Wards. Boletim Goiano de Geografia, v. 28, n. 2, p. 75-88, 2008. https://doi.org/10.5216/bgg.v28i2.5724

Fernandes, D. S.; Kruger, L. F.; Heinemann, A. B.; Rocha, R. P. Identificação e caracterização de ambientes homogêneos de eventos de seca/umidade com base em simulações climáticas regionais. Bragantia, v. 71, n. 2, p. 290-298, 2012.

Hair Jr., J. F.; Black, W. C.; Babin, B. J.; Anderson, R. E.; Tatham, R. L. Análise multivariada de dados. 6. ed. São Paulo: Bookman, 2009.

IEA - International Agency of Energy. Snapshot of Global Photovoltaic Markets. 2018. Disponível em: <http://www.iea-pvps.org/fileadmin/dam/public/report/statistics/IEA-PVPS_-_A_Snapshot_ of_Global_PV_-_1992-2017.pdf>. Acesso em: 16 fev. 2019.

Jannuzzi, P. M.; Carlo, S. Da agenda de desenvolvimento do milênio ao desenvolvimento sustentável: oportunidades e desafios para planejamento e políticas públicas no século XXI. Bahia Análise \& Dados, v. 28, n. 2, p. 6-27, 2019.

Keller Filho, T.; Assad, E. D.; Lima, P. R. Rainfall homogeneous areas in Brazil. Pesquisa Agropecuária Brasileira, v. 40, n.4, p. 311-322, 2005. https://doi.org/10.1590/S0100204X2005000400001

Kunz, A.; Otenio, M. H.; Leitão, R. C.; Gambetta, R. (Eds.). Energia limpa e acessível: contribuições da Embrapa. Brasília: Embrapa, 2018. (Objetivos do desenvolvimento sustentável; 7).

Lopez, R. A. Energia solar para produção de eletricidade. São Paulo: Artliber, 2012.

Machado, C. T.; Miranda, F. S. Energia solar fotovoltaica: uma breve revisão. Revista Virtual de Química, v. 7, n. 1, p. 126-143, 2014. https://doi.org/10.5935/1984-6835.20150008

Martins, G. A.; Domingues, O. Estatística geral e aplicada. São Paulo: Atlas, 2017.

Massini-Cacliari, G.; Domingues, M. A. C.; Vidotti, S. A. B. G.; Putti, F. F.; Barreiro, I. M. F.; Buccelli, R. L. O objetivo faz o método: perspectivas para o cálculo do índice anual de evasão da UNESP. In: Manchope, E. C. P.; Araújo, A.; Miranda, D. L.; Costa, F. G.; Massini-Cagliari, G.; Fortes, N. L. P.; Wolff, 
P. S.; Luz, S. C. T.; Maquêa, S. C. T. Interiorização do ensino superior: protagonismo das universidades estaduais e municipais no desenvolvimento regional. Cascavel: Edunioeste, 2018. p. 127-140. Disponível em: <https://www5.unioeste.br/portalunioeste/images/Editora/abruem2018.pdf\#page=127>. Acesso em: 16 fev. 2019.

Minha Casa Solar. L\&F Comércio, Projetos e Consultoria LTDA. Disponível em: <https://www.minhacasasolar.com.br>. Acesso em: 07 jul. 2018.

Nascimento, F. A.; Vieira, S. A.; Andrade Júnior, S. J.; Cavalcanti, L. A. P. Integração de um sistema fotovoltaico isolado e de coleta seletiva de resíduos em um quiosque multifuncional. Revista Brasileira de Gestão Ambiental e Sustentabilidade, v. 2, n. 2, p. 43-50, 2015.

ONU - Organização das Nações Unidas. Agenda 2030 para o desenvolvimento sustentável. 2015. Disponível em: <https://nacoesunidas.org/tema/agenda2030/>. Acesso em: 09 maio 2019.

Pereira, E. B.; Martins, F. R.; Abreu, S. L.; Rüther, R. Atlas brasileiro de energia solar. São José dos Campos: INPE, 2006. v. 1.

Reis, L. B. Geração de energia elétrica: tecnologia, inserção ambiental, planejamento, operação e análise de viabilidade. São Paulo: Manole, 2015.

Rüther, R.; Salamoni, I. O potencial dos setores urbanos brasileiros para a geração de energia solar fotovoltaica de forma integrada às edificações. Fórum Patrimônio: Ambiente Construído e Patrimônio Sustentável, v. 4, n. 1, p. 1-17, 2013.

São Paulo. Secretaria de Energia. Subsecretaria de Energias Renováveis. Energia solar paulista: levantamento do potencial. 2006.2 Disponível em: <http://dadosenergeticos.energia.sp.gov.br/portalcev2/intranet/BiblioVirtual/renovaveis/atlas_e nergia_solar.pdf>. Acesso em: 01 jul. 2018.

Silva, G.; Romero, M. Sustentabilidade urbana aplicada: Análise dos processos de dispersão, densidade e uso e ocupação do solo para a Cidade de Cuiabá, Estado de Mato Grosso, Brasil. EURE Revista Latinoamericana de Estudios Urbano Regionales, v. 41, n. 122, p. 209-237, 2015. https://doi.org/10.4067/S0250-71612015000100010

Silva, J. S.; Ferreira, A. H. R.; Zukowski Jr., J. C. Z. Estudo de viabilidade econômica de Microgeração de energia solar integrada à rede na UFT Campus Gurupi-TO. Revista Cereus, v. 9, n. 1, p. 88-105, 2017.

Silva, R. M. D. Energia solar no Brasil: dos incentivos aos desafios. Brasília: Senado Federal, 2015. Disponível em: <http://www2.senado.leg.br/bdsf/bitstream/handle/id/507212/TD166RutellyMSilva.pdf>. Acesso em: 07 jul. 2019.

Strangueto, K. M. Estimativa do potencial brasileiro de produção de energia elétrica através de sistemas fotovoltaicos flutuantes em reservatórios de hidroelétricas. Campinas: Universidade Estadual de Campinas, 2016. (Tese de Doutorado).

UNESP - Universidade Estadual Paulista. Anuário Estatístico. 2016. Disponível em: <https://www2.unesp.br/portal\#!/unidades>. Acesso: 29 jun. 2018.

Informação da Licença: Este é um artigo Open Access distribuído sob os termos da Licença Creative Commons Attribution, que permite uso irrestrito, distribuição e reprodução em qualquer meio, desde que a obra original seja devidamente citada. 